\title{
Low-Temperature Properties of the Quantum Heisenberg Antiferromagnet on Some One-Dimensional Lattices Containing Equilateral Triangles
}

\author{
M. MAKSymenko ${ }^{a}$, O. DerzhKO $^{a}$ And J. Richter ${ }^{b}$ \\ ${ }^{a}$ Institute for Condensed Matter Physics, NAS of Ukraine, 1 Svientsitskii Street, L'viv-11, 79011, Ukraine \\ ${ }^{b}$ Institut für Theoretische Physik, Universität Magdeburg, P.O. Box 4120, 39016 Magdeburg, Germany
}

(Received November 9, 2010)

\begin{abstract}
We consider the quantum Heisenberg antiferromagnet in a magnetic field on two one-dimensional lattices containing equilateral triangles (a chain of corner-sharing double tetrahedra and a frustrated three-leg ladder) which support localized-magnon states. By mapping of the localized-magnon degrees of freedom on a classical lattice gas we obtain high-field thermodynamic quantities of the models at low temperatures.
\end{abstract}

PACS: 75.10.Jm, 75.40.Gb

\section{Introduction}

Recently it has been shown how the low-temperature thermodynamics of a large class of geometrically frustrated quantum Heisenberg antiferromagnets in magnetic fields can be studied using the concept of localized-magnon states [1], for a review see Ref. [2]. For this class of models the lowest-energy states in the one-magnon subspace are the so-called localized-magnon states, i.e., the spin excitations above the fully polarized ferromagnetic state are localized on a small part of the lattice (trapping cell). Moreover, independent (isolated) localized magnons are the lowest-energy states in many-magnon subspaces. Their degeneracy can be calculated by mapping on corresponding classical lattice gases and, as a result, the contribution of localized magnons to thermodynamics can be estimated. In extension to these previous studies we consider here lattices with triangular traps, which have not been considered so far. As it is shown below, such triangular traps may produce new features, since the localized-magnon state trapped on a triangle possesses an extra degree of freedom, namely the chirality. Thus, an extra degeneracy of independent localized-magnon states becomes relevant for the low-temperature physics.

\section{The model}

To be specific, we consider the standard quantum $(s=1 / 2)$ Heisenberg antiferromagnet with the Hamiltonian

$$
H=\sum_{(n m)} J_{n m} \boldsymbol{s}_{n} \cdot \boldsymbol{s}_{m}-h S^{z}, \quad S^{z}=\sum_{n} s_{n}^{z}
$$

on the two one-dimensional lattices shown in Fig. 1 (see, e.g., Refs. $[3,4])$. The first sum in (1) runs over neighboring sites, the second sum in (1) runs over all $N$ lattice sites. $J_{n m}>0$ acquires two values: $J_{2}$ along equilateral triangles (bold bonds in Fig. 1) and $J_{1}$ along all other bonds (thin bonds in Fig. 1). $h \geq 0$ is the external magnetic field. We introduce the number of cells $\mathcal{N}$ which equals $N / 4$ for the double tetrahedra chain and $N / 3$ for the frustrated three-leg ladder. In what follows we construct the ground states in the subspaces with $0 \leq$ $n \leq n_{\max }$ magnons (i.e., for $N / 2-n_{\max } \leq S^{z} \leq N / 2$ ), where $n_{\max }=\mathcal{N}$ for the double tetrahedra chain and $n_{\max }=\mathcal{N} / 2$ for the frustrated three-leg ladder. Furthermore, we calculate the degeneracy of ground states $g_{\mathcal{N}}(n)$ in these subspaces and, as a result, obtain the thermodynamic quantities for both spin models in low-temperature strong-field regime.

\section{Many-magnon states}

We begin with the one-magnon subspace. We find that the twofold degenerate and completely dispersive (flat) one-magnon band $\varepsilon_{1,2}(\kappa)=-J_{1}-3 J_{2} / 2$ (double tetrahedra chain) or $\varepsilon_{1,2}(\kappa)=-3 J_{1}-3 J_{2} / 2$ (frustrated three-leg ladder) becomes the lowest-energy one if $J_{2}>2 J_{1}$. Then the saturation field $h_{1}$ is given by $h_{1}=-\varepsilon_{1,2}(\kappa)$. The corresponding eigenstates (localized magnons) are located along one of $\mathcal{N}$ triangles and they have the energy $E_{\mathrm{FM}}-h_{1}$, where $E_{\mathrm{FM}}$ is the energy of the ferromagnetically polarized state. The twofold degeneracy comes 


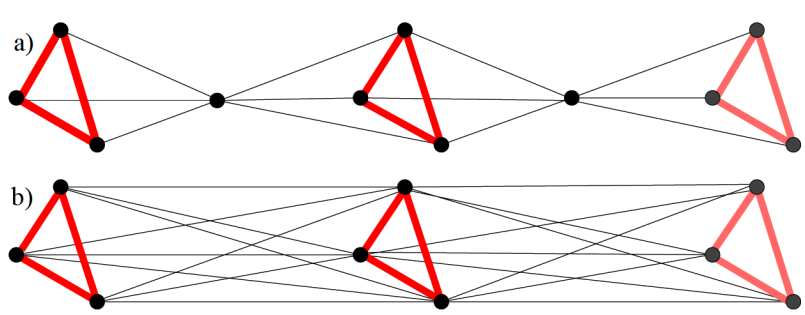

Fig. 1. Double tetrahedra chain (a) and frustrated three-leg ladder (b). Exchange integrals acquire two values: $J_{2}>0$ along equilateral triangle sides (bold) and $J_{1}>0$ along all other bonds (thin).

from the chirality of the triangles.

Next we pass to the $n$-magnon subspace with $1<n \leq$ $n_{\max }$. A many-magnon state which consists of $n$ localized magnons located on different triangular traps (double tetrahedra chain) or on different but not neighboring triangular traps (frustrated three-leg ladder) is the lowest-energy state in the $n$-magnon subspace with the energy $E_{\mathrm{FM}}-n h_{1}$. Importantly, we can easily count the ground-state degeneracy in the $n$-magnon subspace $g_{\mathcal{N}}(n)$. We find $g_{\mathcal{N}}(n)=2^{n} \mathcal{C}_{\mathcal{N}}^{n}$ (double tetrahedra chain) or $g_{\mathcal{N}}(n)=2^{n} \mathcal{Z}(n, \mathcal{N})$, where $\mathcal{Z}(n, \mathcal{N})$ stands for the canonical partition function of $n$ hard dimers on a simple chain of $\mathcal{N}$ sites (frustrated three-leg ladder). Let us note that the factor $2^{n}$ appears in $g_{\mathcal{N}}(n)$ due to the chirality of the triangles (compare with the results for the diamond chain and frustrated two-leg ladder [5]).

\section{Thermodynamics}

Considering the case when a magnetic field $h$ is present we find that the independent localized-magnon states are the lowest-energy states having the energy $E_{\mathrm{FM}}-h N / 2-$ $n\left(h_{1}-h\right)$ and the degeneracy $g_{\mathcal{N}}(n)$. Due to their huge degeneracy the localized-magnon states yield the dominant contribution to the partition function of the spin model (1) at low temperatures $T$ and high fields $h \approx h_{1}$, i.e., $Z(T, h, N) \approx Z_{\operatorname{lm}}(T, h, N)$, where

$$
\begin{gathered}
Z_{\operatorname{lm}}(T, h, N)=\sum_{n=0}^{n_{\max }} g_{\mathcal{N}}(n) \mathrm{e}^{-\frac{E_{\mathrm{FM}}-h \frac{N}{2}-n\left(h_{1}-h\right)}{T}} \\
=\mathrm{e}^{-\frac{E_{\mathrm{FM}}-h \frac{N}{2}}{T}} \Xi(T, \mu, \mathcal{N}) ; \quad \mu=h_{1}-h .
\end{gathered}
$$

(We set $k_{\mathrm{B}}=1$.) The sum in Eq. (2) can be easily evaluated for both lattices: $\Xi(T, \mu, \mathcal{N})=\left(1+2 \mathrm{e}^{\mu / T}\right)^{\mathcal{N}}$ (double tetrahedra chain) and $\Xi(T, \mu, \mathcal{N})=\lambda_{1}^{\mathcal{N}}+\lambda_{2}^{\mathcal{N}}$, $\lambda_{1,2}=1 / 2 \pm \sqrt{1 / 4+2 \mathrm{e}^{\mu / T}}$ (frustrated three-leg ladder). Based on this result the thermodynamic quantities can be easily calculated from the free energy $F_{\operatorname{lm}}(T, h, N)=$ $-T \ln Z_{\operatorname{lm}}(T, h, N)$. For example, the specific heat is given by $C_{\operatorname{lm}}(T, h, N)=-T \partial^{2} F_{\operatorname{lm}}(T, h, N) / \partial T^{2}$.

In Fig. 2 we compare the temperature dependence of localized-magnon contribution $C_{\operatorname{lm}}(T, h, N)$ of the specific heat (lines) and specific heat of the full model

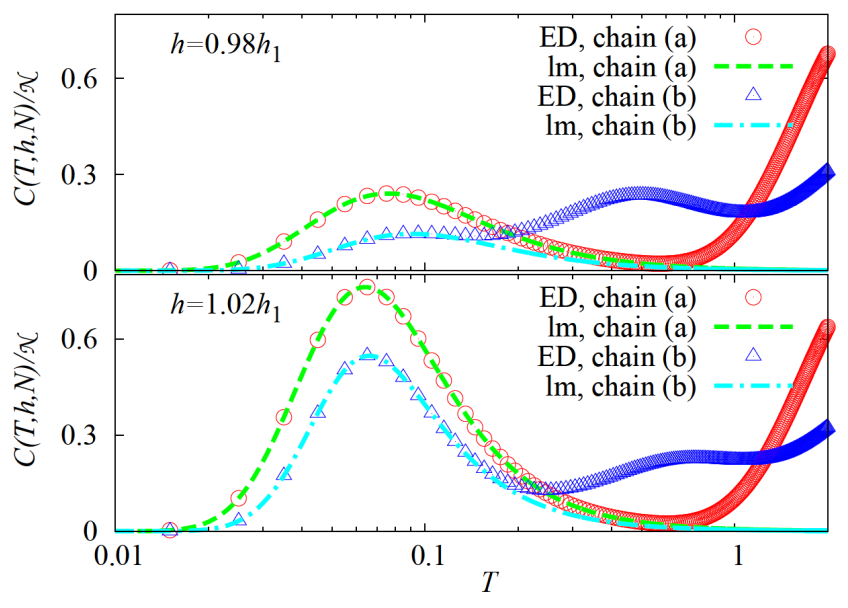

Fig. 2. Specific heat for the double tetrahedra chain and frustrated three-leg ladder which consist of $\mathcal{N}=4$ cells for small deviations from the saturated magnetic field $h=0.98 h_{1}$ and $h=1.02 h_{1}$. Here $J_{1}=1$ and $J_{2}=5$ and hence $h_{1}=17 / 2$ (double tetrahedra chain) or $h_{1}=21 / 2$ (frustrated three-leg ladder). Exact diagonalization data are shown by symbols, localized-magnon predictions are shown by lines.

$C(T, h, N)$ at $h=0.98 h_{1}$ and $h=1.02 h_{1}$ for finite chains (symbols). To perform exact diagonalizations we use the ALPS package [6]. The specific heat shows a well-pronounced low-temperature maximum due to the localized magnons. Obviously the elaborated approach reproduces perfectly well the low-temperature behavior of the specific heat thus illustrating the dominant role of the localized-magnon contribution to thermodynamics at low $T$ and $h$ around $h_{1}$.

\section{Outlook}

Several remarks are in order here. First, the considered spin models, in principle, may have solid-state realizations, see, e.g., Refs. $[7,8]$. Second, for the antiferromagnetic Heisenberg frustrated three-leg ladder it is also possible to take into account a substantial part of low-lying excited states [9]. Third, one may add to spin Hamiltonian (1) small extra terms which remove the degeneracy due to the chirality. Manipulation with chirality attracts much interest nowadays [10-12]. Fourth, the elaborated scheme may be applied to the Hubbard model on the lattices shown in Fig. 1, see Refs. [13-15]. These issues will be discussed in an extended publication.

\section{Acknowledgments}

This paper was presented at the 14th Czech and Slovak Conference on Magnetism CSMAG'10 (July 6-9, 2010, Košice, Slovakia). O.D. thanks Pavol Jozef Šafárik University in Košice for kind hospitality during the conference. 


\section{References}

[1] J. Schulenburg, A. Honecker, J. Schnack, J. Richter, H.-J. Schmidt, Phys. Rev. Lett. 88, 167207 (2002).

[2] O. Derzhko, J. Richter, A. Honecker, H.-J. Schmidt, Fiz. Nizk. Temp. (Kharkiv) 33, 982 (2007); Low Temp. Phys. 33, 745 (2007).

[3] O. Rojas, F.C. Alcaraz, Phys. Rev. B 67, 174401 (2003).

[4] A. Honecker, F. Mila, M. Troyer, Eur. Phys. J. B 15 227 (2000).

[5] O. Derzhko, J. Richter, Eur. Phys. J. B 52, 23 (2006).

[6] F. Albuquerque, F. Alet, P. Corboz, P. Dayal, A. Feiguin, S. Fuchs, L. Gamper, E. Gull, S. Gürtler, A. Honecker, R. Igarashi, M. Körner, A. Kozhevnikov, A. Läuchli, S.R. Manmana, M. Matsumoto, I.P. McCulloch, F. Michel, R.M. Noack, G. Pawłowski, L. Pollet, T. Pruschke, U. Schollwöck, S. Todo, S. Trebst, M. Troyer, P. Werner, S. Wessel, J. Magn. Magn. Mater. 310, 1187 (2007).

[7] G. Seeber, P. Kögerler, B.M. Kariuki, L. Cronin, Chem. Commun., 1580 (2004).
[8] N.B. Ivanov, J. Schnack, R. Schnalle, J. Richter, P. Kögerler, G.N. Newton, L. Cronin, Y. Oshima, H. Nojiri, Phys. Rev. Lett. 105, 037206 (2010).

[9] O. Derzhko, T. Krokhmalskii, J. Richter, Phys. Rev. B 82, 214412 (2010).

[10] M. Trif, F. Troiani, D. Stepanenko, D. Loss, Phys. Rev. Lett. 101, 217201 (2008).

[11] L.N. Bulaevskii, C.D. Batista, M.V. Mostovoy, D.I. Khomskii, Phys. Rev. B 78, 024402 (2008).

[12] B. Georgeot, F. Mila, arXiv:0902.4652.

[13] C.D. Batista, B.S. Shastry, Phys. Rev. Lett. 91, 116401 (2003).

[14] A. Honecker, J. Richter, Condens. Matter Phys. (L'viv) 8, 813 (2005).

[15] O. Derzhko, A. Honecker, J. Richter, Phys. Rev. B 76, 220402(R) (2007); 79, 054403 (2009); O. Derzhko, J. Richter, A. Honecker, M. Maksymenko, R. Moessner, Phys. Rev. B 81, 014421 (2010). 